\title{
A NOTE ON THE PERIODIC ORBITS OF A KIND OF DUFFING EQUATIONS
}

\author{
JAUME LLIBRE ${ }^{1}$ AND ANA RODRIGUES ${ }^{2}$
}

\begin{abstract}
We study the periodic orbits of the modified Duffing differential equation $\ddot{y}+a y-\varepsilon y^{3}=\varepsilon h(y, \dot{y})$, with $a>0, \varepsilon$ a small parameter and $h$ a $\mathcal{C}^{2}$ function in its variables.
\end{abstract}

\section{INTRODUCTION}

In a paper published in 1922, Hamel [7] provided the first general results for the existence of periodic solutions of the periodically forced pendulum equation

$$
\ddot{y}+a \sin y=b \sin t \text {. }
$$

This equation was the main subject of a monograph published four years earlier by Duffing [5], who had restricted his study to the approximate determination of the periodic solutions for the following approximation of equation (1):

$$
\ddot{y}+a y-c y^{3}=b \sin t,
$$

which now is known as the Duffing differential equation. For more details on the history of these differential equations see the paper of Mawhin [9]. Many of the 190 references quoted in this last paper are on the periodic orbits of different kind of Duffing equations, and from its publication many new papers working on these type of periodic orbits also have been published, see for instance the papers $[3,4,12]$ and the quoted references in there.

Here we consider the following modified Duffing differential equation

$$
\ddot{y}+a y-\varepsilon y^{3}=\varepsilon h(y, \dot{y}),
$$

with $a>0, \varepsilon$ a small parameter and $h$ a $\mathcal{C}^{2}$ function in its variables.

We recall that $\bar{k}$ is a simple zero of a real function $f(k)$ if $f(\bar{k})=0$ and $(d f / d k)(\bar{k}) \neq 0$.

Our main result is the following.

Theorem 1. For $\varepsilon \neq 0$ sufficiently small and for every simple positive zero $\bar{k}$ of the function

$$
f(k)=\int_{0}^{2 \pi} h\left(k \cos \theta, \frac{k \sin \theta}{\sqrt{a}}\right) \cos \theta d \theta,
$$

the modified Duffing differential equation (2) has a periodic orbit $y(t)$ such that when $\varepsilon \rightarrow 0$ we have that $(\dot{y}(t), y(t))$ tends to the periodic orbit given by the ellipse

$$
\frac{x^{2}}{\bar{k}^{2}}+\frac{y^{2}}{\bar{k}^{2} / a}=1,
$$

of the differential system $\dot{x}=-a y, \dot{y}=x$. 
Theorem 1 will be proved in section 3 using the averaging theory, see section 2 where is described the result on averaging theory that we shall need to use here. In general the main difficulty for studying the existence of periodic orbits using the averaging theory is to find a change of variables which write the studied differential system into the normal form of the averaging theory (see (4)).

Now we shall do some applications of Theorem 1.

Corollary 2. Let $h(x, y)=\sin x$. Then for every positive integer $n$ there exists an $\varepsilon_{0}>0$ sufficiently small such that for all $\varepsilon \in\left(-\varepsilon_{0}, \varepsilon_{0}\right)$ the modified Duffing differential equation (2) has at least $n$ periodic orbits.

Corollary 3. Let $h(x, y)=\sum_{i+j=0}^{3} a_{i j} x^{i} y^{j}$ be an arbitrary polynomial of degree 3 . If $a_{10}\left(a_{12}+3 a a_{30}\right)<0$, then the modified Duffing differential equation (2) has at least one periodic orbit $y(t)$ such that when $\varepsilon \rightarrow 0$ we have that $(\dot{y}(t), y(t))$ tends to the periodic orbit of the differential system $\dot{x}=-a y, \dot{y}=x$, given by the ellipse (3) with

$$
k=2 \sqrt{-\frac{a a_{10}}{a_{12}+3 a a_{30}}} .
$$

Corollaries 2 and 3 are proved in section 4 .

\section{BASIC RESUlts}

In this section we present the basic result from the averaging theory that we shall need for proving the main result of this paper. For a general introduction to the averaging theory and related topics see the books $[2,6,8,10,11]$.

We consider the differential system

$$
\dot{\mathbf{x}}=\varepsilon F(t, \mathbf{x})+\varepsilon^{2} R(t, \mathbf{x}, \varepsilon),
$$

with $\mathbf{x} \in U \subset \mathbb{R}^{n}, U$ a bounded domain and $t \geq 0$. Moreover, we assume that $F(t, \mathbf{x})$ and $R(t, \mathbf{x}, \varepsilon)$ are $T$-periodic in $t$.

The averaged system associated to system (4) is defined by

$$
\dot{\mathbf{y}}=\varepsilon f(\mathbf{y})
$$

where

$$
f(\mathbf{y})=\frac{1}{T} \int_{0}^{T} F(s, \mathbf{y}) d s .
$$

The next theorem says us under which conditions the singular points of the averaged system (5) provide $T$-periodic orbits of system (4). For a proof see Theorem 2.6.1 of [10], Theorems 11.5 and 11.6 of [11], and Theorem 4.1.1 of [6].

Theorem 4. We consider system (4) and assume that the vector functions $F, R$, $D_{\mathbf{x}} F, D_{\mathbf{x}}^{2} F$ and $D_{\mathbf{x}} R$ are continuous and bounded by a constant $M$ (independent of $\varepsilon$ ) in $[0, \infty) \times U$ with $-\varepsilon_{0}<\varepsilon<\varepsilon_{0}$. Moreover we suppose that $F$ and $R$ are $T$-periodic in $t$, with $T$ independent of $\varepsilon$.

(a) If $a \in U$ is a singular point of the averaged system (5) such that $\operatorname{det}\left(D_{\mathbf{x}} f(a)\right) \neq$ 0 then, for $|\varepsilon|>0$ sufficiently small there exists a unique $T$-periodic solution $\mathbf{x}_{\varepsilon}(t)$ of system (4) such that $\mathbf{x}_{\varepsilon}(0) \rightarrow a$ as $\varepsilon \rightarrow 0$. 
(b) If the singular point a of the averaged system (5) is hyperbolic then, for $|\varepsilon|>0$ sufficiently small the corresponding periodic solution $\mathbf{x}_{\varepsilon}(t)$ of system (4) is hyperbolic and of the same stability type than a.

\section{Proof of Theorem 1}

We write the second order differential equation (2) as the system of two differential equations of first order

$$
\begin{aligned}
& \dot{x}=-a y+\varepsilon\left(y^{3}+h(x, y)\right), \\
& \dot{y}=x .
\end{aligned}
$$

Now we shall study the solution of the differential system (7) with $\varepsilon=0$.

Lemma 5. With the exception of the singular point located at the origin of coordinates, the differential system (7) with $\varepsilon=0$ has all its orbits periodic leaving on the ellipses

with $k$ real and positive.

$$
\frac{x^{2}}{\bar{k}^{2}}+\frac{y^{2}}{\bar{k}^{2} / a}=1
$$

Proof. Assuming $a>0$, the general solution of the system (7) with $\varepsilon=0$ and with initial conditions $x(0)=x_{0}, y(0)=y_{0}$ is

$$
x(t)=x_{0} \cos (\sqrt{a} t)-\sqrt{a} y_{0} \sin (\sqrt{a} t),
$$

$$
y(t)=\frac{\sqrt{a} y_{0} \cos (\sqrt{a} t)+x_{0} \sin (\sqrt{a} t)}{\sqrt{a}} .
$$

All the orbits of the system (7) with $\varepsilon=0$ with exception of the origin are periodic of period $2 \pi / \sqrt{a}$.

We solve (8) with respect to $\cos (\sqrt{a} t)$ and $\sin (\sqrt{a} t)$ and we get

$$
\begin{aligned}
& \cos (\sqrt{a} t)=\frac{-x x_{0}-a y y_{0}}{x_{0}^{2}+a y_{0}^{2}}, \\
& \sin (\sqrt{a} t)=\frac{-\sqrt{a} x_{0} y+\sqrt{a} x y_{0}}{x_{0}^{2}+a y_{0}^{2}} .
\end{aligned}
$$

using the trigonometric relation $\sin ^{2}(x)+\cos ^{2}(x)=1$ we get after simplifying

$$
\frac{x^{2}+a y^{2}}{x_{0}^{2}+a y_{0}^{2}}=1
$$

Thus, we get the ellipsis in the statement of Lemma 5 with $\bar{k}^{2}=x_{0}^{2}+a y_{0}^{2}$.

Proof of Theorem 1. Recall Lemma 5. The solution of system (7) with $\varepsilon=0$ is the ellipsis

$$
\frac{x^{2}}{x_{0}^{2}+a y_{0}^{2}}+\frac{y^{2}}{\left(x_{0}^{2} / a+y_{0}^{2}\right)}=1,
$$

centered at the origin with semi-major axis on the $x$-axis and semi-minor axis on the $y$-axis. The semi-major axis is equal to $\sqrt{x_{0}^{2}+a y_{0}^{2}}$ and the semi-minor axis is equal 
to $\sqrt{\left(x_{0}^{2}+a y_{0}^{2}\right) / a}$. So we write the original system of two differential equations of first order ( 7$)$ in elliptic coordinates. We do the change of coordinates

$$
x=\sqrt{x_{0}^{2}+a y_{0}^{2}} r \cos \theta, y=\sqrt{x_{0}^{2} / a+y_{0}^{2}} r \sin \theta .
$$

In these new coordinates, system (7) becomes

$$
\begin{aligned}
& \dot{r}=\frac{\varepsilon \cos \theta}{k}\left[h\left(r k \cos \theta, r \frac{k}{\sqrt{a}} \sin \theta\right)+r^{3} k^{3} \sin ^{3} \theta\right], \\
& \dot{\theta}=\sqrt{a}-\frac{\varepsilon \sin \theta}{r k}\left[h\left(r k \cos \theta, r \frac{k}{\sqrt{a}} \sin \theta\right)+r^{3} k^{3} \sin ^{3} \theta\right] .
\end{aligned}
$$

where $k=\sqrt{x_{0}^{2}+a y_{0}^{2}}$.

Now applying Theorem 4 of the averaging theory described in Section 2 to system (10) and since

$$
\int_{0}^{2 \pi} \frac{\cos \theta}{k / r}\left[k^{3} \sin ^{3} \theta\right] d \theta=0,
$$

it follows immediately Theorem 1.

\section{Applications}

In these section we shall prove the corollaries.

Proof of Corollary 2. We apply Theorem 1 to system (7) with

$$
h(x, y)=b \sin x \text {. }
$$

We write the second order differential equation as the system of two differential equations of first order

$$
\begin{aligned}
& \dot{x}=-a y+\varepsilon\left(y^{3}+b \sin x\right), \\
& \dot{y}=x .
\end{aligned}
$$

We write system (11) in elliptic coordinates

$$
x=r \cos (\theta), y=r \sin \theta / \sqrt{a}
$$

and we get the system in the new coordinates $(r, \theta)$ given by

$$
\begin{aligned}
& \dot{r}=\varepsilon \cos \theta\left(\frac{r^{3} \sin ^{3} \theta}{a^{3 / 2}}+b \sin t\right), \\
& \dot{\theta}=\sqrt{a}-\frac{\varepsilon\left(\frac{r^{3} \sin ^{4} \theta}{a^{3 / 2}}+b \sin \theta \sin t\right)}{r} .
\end{aligned}
$$

The system of equations (12) with $\varepsilon=0$ and initial conditions $r(0)=r_{0}, \theta(0)=\theta_{0}$ has the solutions

$$
r(t)=r_{0}, \theta(t)=\sqrt{a} t+\theta_{0},
$$

this is, all orbits with $r_{0}>0$ are periodic of period $2 \pi / \sqrt{a}$.

We obtain after some tedious computations that the function $\left(f_{1}, f_{2}\right)$ are given by

$$
\begin{aligned}
& f_{1}=b\left(-2 \cos \theta_{0} \sin ^{2}\left(\frac{\pi}{\sqrt{a}}\right)+\sqrt{a} \sin \left(\frac{\pi}{\sqrt{a}}\right) \sin \theta_{0}\right), \\
& f_{2}=3(-1+a) \pi r_{0}^{3}-4 a^{5 / 2} b \cos \theta_{0} \sin \left(\frac{2 \pi}{\sqrt{a}}\right)-8 a^{2} b \sin ^{2}\left(\frac{\pi}{\sqrt{a}}\right) \sin \theta_{0} .
\end{aligned}
$$


Now solving $f_{1}=0$ with respect to $\theta_{0}$ we get

$$
\theta_{0}^{ \pm}= \pm \arccos \left[\frac{\sqrt{a} \sin \left(\frac{2 \pi}{\sqrt{a}}\right)}{\sqrt{4 \sin ^{4}\left(\frac{\pi}{\sqrt{a}}\right)+a \sin ^{2}\left(\frac{2 \pi}{\sqrt{a}}\right)}}\right] .
$$

Substituting $\theta_{0}^{-}$in $f_{2}=0$ and simplifying we get

$$
f_{2}=3(a-1) \pi r_{0}^{3}+A,
$$

where

$A=-4 a^{2} b\left[\frac{a \sin ^{2}\left(\frac{2 \pi}{\sqrt{a}}\right)}{\sqrt{4 \sin ^{4}\left(\frac{\pi}{\sqrt{a}}\right)+a \sin ^{2}\left(\frac{2 \pi}{\sqrt{a}}\right)}}\right]-2 \sqrt{\frac{1}{a+(1-a) \sin ^{2}\left(\frac{\pi}{\sqrt{a}}\right)}}\left|\sin ^{3}\left(\frac{\pi}{\sqrt{a}}\right)\right|$.

Thus if $A(a-1)<0$, we have a positive solution for $r_{0}$.

Now substituting $\theta_{0}^{+}$in $f_{2}=0$ and simplifying we get

$$
f_{2}=3(a-1) \pi r_{0}^{3}+B,
$$

where

$B=-4 a^{2} b\left[\frac{a \sin ^{2}\left(\frac{2 \pi}{\sqrt{a}}\right)}{\sqrt{4 \sin ^{4}\left(\frac{\pi}{\sqrt{a}}\right)+a \sin ^{2}\left(\frac{2 \pi}{\sqrt{a}}\right)}}\right]+2 \sqrt{\frac{1}{a+(1-a) \sin ^{2}\left(\frac{\pi}{\sqrt{a}}\right)}}\left|\sin ^{3}\left(\frac{\pi}{\sqrt{a}}\right)\right|$.

Thus if $B(a-1)<0$, we have a positive solution for $r_{0}$. Now $A$ and $B$ have the same sign (and consequently there are two periodic orbits) if the following expression is positive:

$$
-\frac{4 \sin ^{6}\left(\frac{\pi}{\sqrt{a}}\right)}{a-(-1+a) \sin ^{2}\left(\frac{\pi}{\sqrt{a}}\right)}+\frac{a^{2} \sin ^{4}\left(\frac{2 \pi}{\sqrt{a}}\right)}{4 \sin ^{4}\left(\frac{\pi}{\sqrt{a}}\right)+a \sin ^{2}\left(\frac{2 \pi}{\sqrt{a}}\right)} .
$$

We analyze in Figure 1 the sign of this expression. Note that it takes positive and negative values.

In fact, we must check that both solutions when $A B>0$ do not correspond to different initial conditions of the same periodic orbit.

We solve equation (14) with respect to $r_{0}$ and we get two different solutions

$$
r=\frac{B^{1 / 3}}{(3 \pi)^{1 / 3}(1-a)^{1 / 3}}, \theta=\arccos \left[\frac{\sqrt{a} \sin \left(\frac{2 \pi}{\sqrt{a}}\right)}{\sqrt{4 \sin ^{4}\left(\frac{\pi}{\sqrt{a}}\right)+a \sin ^{2}\left(\frac{2 \pi}{\sqrt{a}}\right)}}\right]
$$




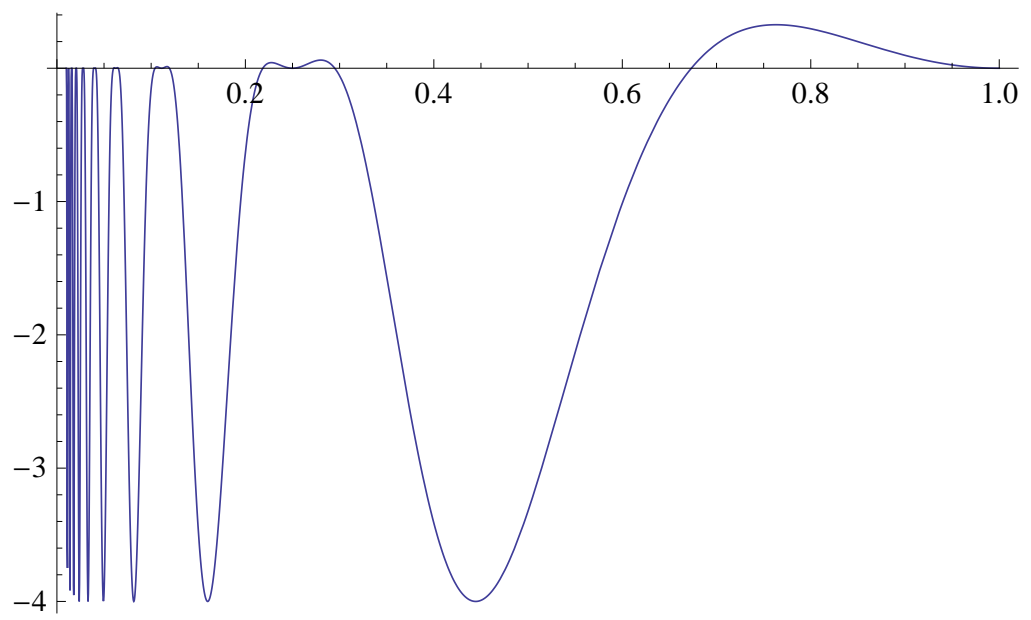

Figure 1. Plot of expression (15) as a function of $a$.

and

$$
r=\frac{A^{1 / 3}}{(3 \pi)^{1 / 3}(1-a)^{1 / 3}}, \theta=-\arccos \left[\frac{\sqrt{a} \sin \left(\frac{2 \pi}{\sqrt{a}}\right)}{\sqrt{4 \sin ^{4}\left(\frac{\pi}{\sqrt{a}}\right)+a \sin ^{2}\left(\frac{2 \pi}{\sqrt{a}}\right)}}\right] .
$$

Substituting the values for $r$ and $\theta$ we got in (16) in the expression for $x_{0}$ and $y_{0}$

$$
x_{0}=r \cos \theta, y_{0}=r \sin \theta / \sqrt{a}
$$

holds

(19)

$$
x_{0}=\frac{\sqrt{a} B^{1 / 3} \sin \left(\frac{2 \pi}{\sqrt{a}}\right)}{(1-a)^{1 / 3}(3 \pi)^{1 / 3} \sqrt{4 \sin ^{4}\left(\frac{\pi}{\sqrt{a}}\right)+a \sin ^{2}\left(\frac{2 \pi}{\sqrt{a}}\right)}}
$$

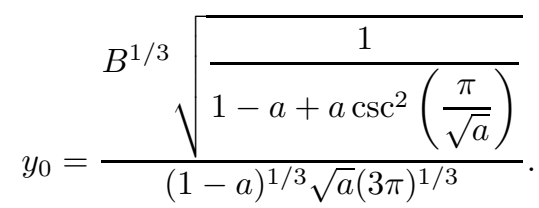


Substituting now the values for $r$ and $\theta$ we got in (17) in the expression for $x_{0}$ and $y_{0}$ given by (18) we get

(20)

$$
x_{0}=\frac{\sqrt{a} A^{1 / 3} \sin \left(\frac{2 \pi}{\sqrt{a}}\right)}{(1-a)^{1 / 3}(3 \pi)^{1 / 3} \sqrt{4 \sin ^{4}\left(\frac{\pi}{\sqrt{a}}\right)+a \sin ^{2}\left(\frac{2 \pi}{\sqrt{a}}\right)}},
$$

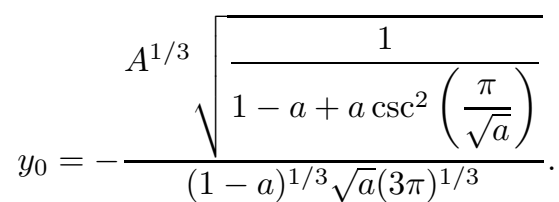

Consider the semi-axis

$$
x_{0}^{2}+a y_{0}^{2}
$$

of the ellipsis. Restricted to the solution we found in (19) holds

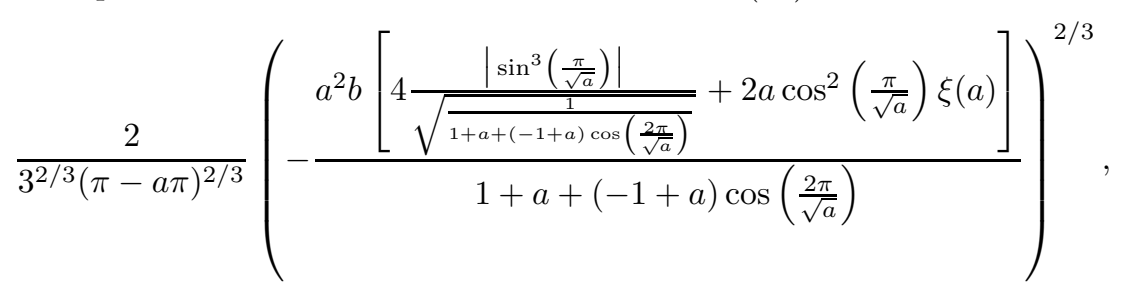

where

$$
\xi(a)=\sqrt{3+a-4 \cos \left(\frac{2 \pi}{\sqrt{a}}\right)-(-1+a) \cos \left(\frac{4 \pi}{\sqrt{a}}\right)},
$$

and restricted to the solution given by $(20)$ we get

$$
\frac{2}{3^{2 / 3}(\pi-a \pi)^{2 / 3}}\left(-\frac{a^{2} b\left[-4 \frac{\left|\sin ^{3}\left(\frac{\pi}{\sqrt{a}}\right)\right|}{\left.\sqrt{\frac{1}{1+a+(-1+a) \cos \left(\frac{2 \pi}{\sqrt{a}}\right)}}+2 a \cos ^{2}\left(\frac{\pi}{\sqrt{a}}\right) \xi(a)\right]}\right.}{1+a+(-1+a) \cos \left(\frac{2 \pi}{\sqrt{a}}\right)}\right)^{2 / 3} .
$$

Clearly there are two different periodic orbits when both exist and for $a \rightarrow 1$ they tend to the same orbit, note that

$$
\lim _{a \rightarrow 1} \frac{a \sin ^{2}\left(\frac{2 \pi}{\sqrt{a}}\right)}{\sqrt{4 \sin ^{4}\left(\frac{\pi}{\sqrt{a}}\right)+a \sin ^{2}\left(\frac{2 \pi}{\sqrt{a}}\right)}}=0 .
$$

Moreover, we have $a+(1-a) \sin ^{2}\left(\frac{\pi}{\sqrt{a}}\right)>0$ if $a>0$. Indeed, we plot in figures 2,3 and 4 the function $a+(1-a) \sin ^{2}\left(\frac{\pi}{\sqrt{a}}\right)$ for different ranges of $a$.

Assume now that $a=1$. We get after a simple computation that $f_{1}$ and $f_{2}$ are given by

$$
f_{1}=-\frac{1}{2} b \sin \theta_{0}, f_{2}=-\frac{3 r_{0}^{3}+4 b \cos \theta_{0}}{8 r_{0}} .
$$




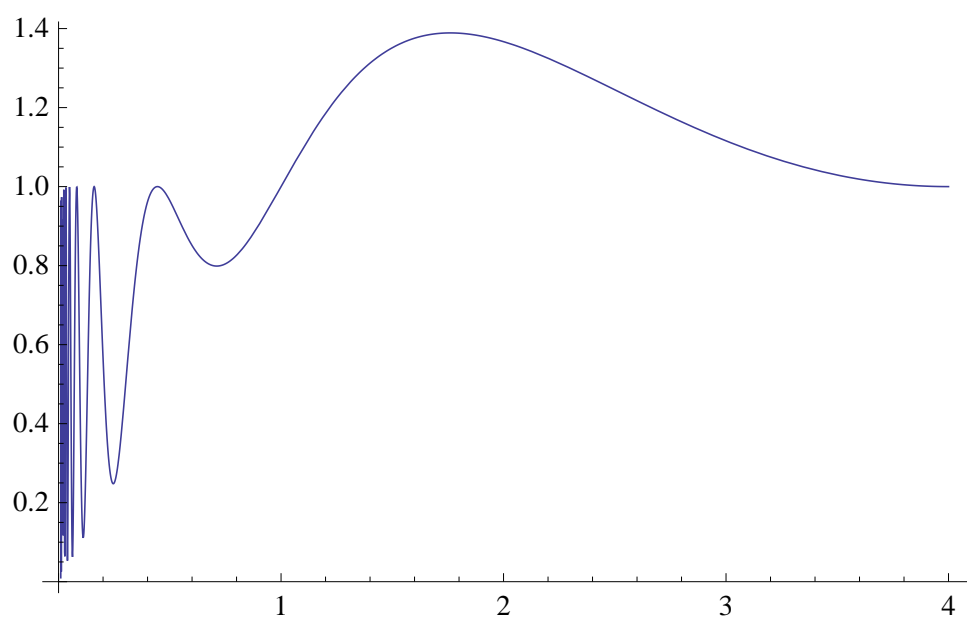

Figure 2. Plot of expression $a+(1-a) \sin ^{2}\left(\frac{\pi}{\sqrt{a}}\right)$ as a function of $a$ for $a \in[0.01,4]$.

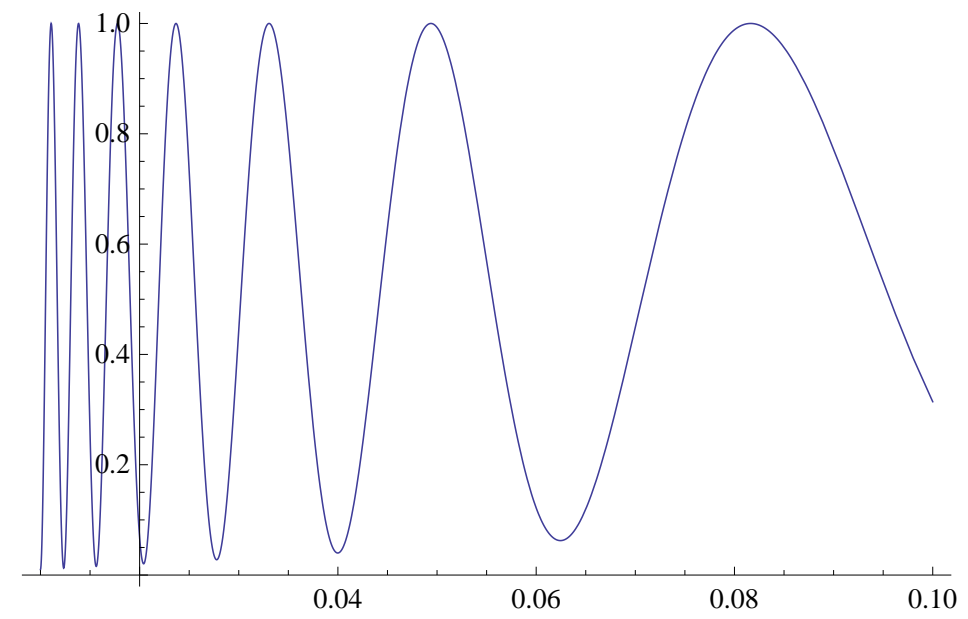

Figure 3. Plot of expression $a+(1-a) \sin ^{2}\left(\frac{\pi}{\sqrt{a}}\right)$ as a function of $a$ for $a \in[0.01,0.1]$.

Thus, we have one positive solution if $b<0$ and one positive solution if $b>0$. Now substituting $x_{0}=r \cos \theta, y_{0}=r \sin \theta / \sqrt{a}$ with $r=(4|b| / 3)^{1 / 3}$ and $\theta=0$ or $\theta=\pi$ in $x_{0}^{2}+a y_{0}^{2}$ holds $\frac{2^{4 / 3}|b|^{2 / 3}}{3^{2 / 3}}$. This finishes the proof of Corollary 2 .

Proof of Corollary 3. We apply Theorem 1 to system (7) with

$h(x, y)=a_{00}+a_{10} x+a_{01} y+a_{20} x^{2}+a_{11} x y+a_{02} y^{2}+a_{30} x^{3}+a_{21} x^{2} y+a_{12} x y^{2}+a_{03} y^{3}$. 


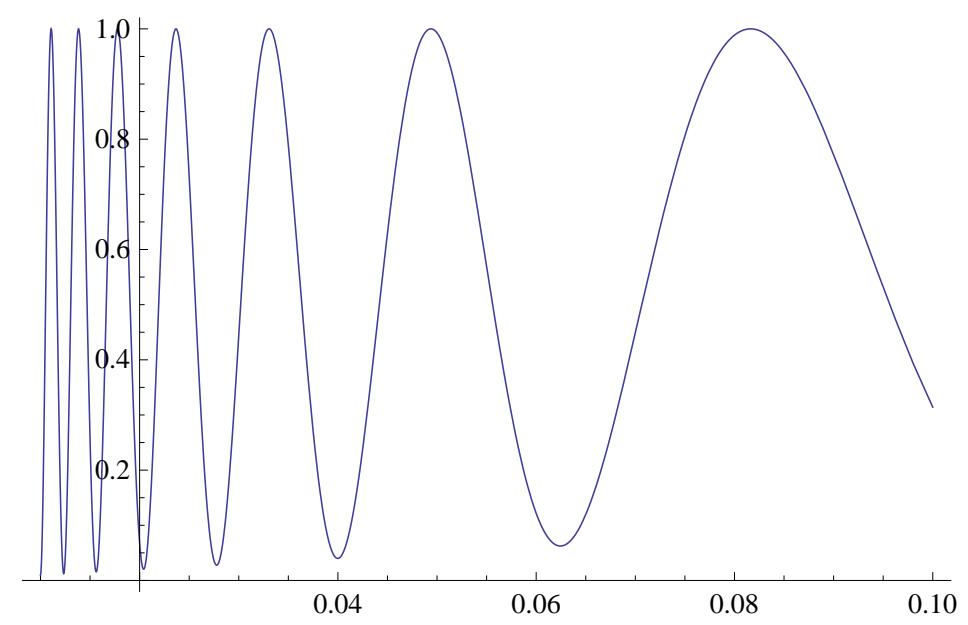

Figure 4. Plot of expression $a+(1-a) \sin ^{2}\left(\frac{\pi}{\sqrt{a}}\right)$ as a function of $a$ for $a \in[1,10]$.

After some simple computation we obtain that the function $f(k)$ is given by

$$
f(k)=\frac{1}{8} k\left(4 a_{10}+\frac{\left(a_{12}+3 a a_{30}\right) k^{2}}{a}\right) .
$$

Solving $f(k)=0$ with respect to $k$ holds

$$
k=0, k=-2 \sqrt{-\frac{a a_{10}}{a_{12}+3 a a_{30}}}, k=2 \sqrt{-\frac{a a_{10}}{a_{12}+3 a a_{30}}},
$$

and this finishes the proof.

\section{ACKNOWLEDGEMENTS}

The first author has been supported by the grants MCYT/FEDER MTM200803437, CIRIT-Spain 2009SGR 410 and ICREA Academia. The second author was supported by FCT Grant BPD/36072/2007. Research of AR supported in part by Centro de Matemática da Universidade do Porto (CMUP) financed by FCT through the programmes POCTI and POSI, with Portuguese and European Community structural funds.

\section{REFERENCES}

[1] M. Abramowitz and I.A. Stegun, Bessel Functions $J$ and $Y, \S 9.1$ in Handbook of Mathematical Functions with Formulas, Graphs, and Mathematical Tables, 9th printing. New York: Dover, pp. 358-364, 1972.

[2] V.I. Arnold, V.V. Kozlov and A.I. Neishtadt, Mathematical aspects of classical and celestial mechanics, Second Printing, Springer-Verlag, Berlin, 1997.

[3] H. Chen And Y. LI, Stability and exact multiplicity of periodic solutions of Duffing equations with cubic nonlinearities, Proc. Amer. Math. Soc. 135 (2007), 3925-3932

[4] H. Chen AND Y. LI, Bifurcation and stability of periodic solutions of Duffing equations, Nonlinearity 21 (2008), 2485-2503.

[5] G. DufFING, Erzwungen Schwingungen bei veränderlicher Eigenfrequenz und ihre technisch Bedeutung, Sammlung Vieweg Heft 41/42, Vieweg, Braunschweig, 1918. 
[6] J. Guckenheimer And P. Holmes, Nonlinear oscillations, dynamical systems, and bifurcation of vector fields, Springer, 1983.

[7] G. HAMEL, Ueber erzwungene Schingungen bei endlischen Amplituden, Math. Ann. 86 (1922), $1-13$.

[8] P. Lochak And C. Meunier, Multiphase averaging for classical systems, Appl. Math. Sciences 72, Springer-Verlag, New York, 1988.

[9] J. Mawhin, Seventy-five years of global analysis around the forced pendulum equation, Proceedings of Equadiff 9 CD rom, Brno 1997, Masaryk University, pp 115-145.

[10] J.A. Sanders And F. Verhulst, Averaging Methods in Nonlinear Dynamical Systems, Applied Mathematical Sci. 59, Springer-Verlag, New York, 1985.

[11] F. Verhulst, Nonlinear Differential Equations and Dynamical Systems, Universitext, Springer-Verlag, Berlin, 1996.

[12] X. Wu, J. Li And Y, Zhou, A priori bounds for periodic solutions of a Duffing equation, J. Appl. Math. Comput. 26 (2008), 535-543.

1 Departament de Matemàtiques, Universitat Autònoma de Barcelona, Bellaterra, 08193 Barcelona, Catalonia, Spain.

E-mail address: jllibre@mat.uab.cat

2 Centro de Matemática da Universidade do Porto (CMUP), Rua do Campo Alegre 687, 4169-007 Porto, Portugal.

E-mail address: arodrig@math.iupui.edu 